\title{
Characteristics Associated with Survival in Patients Receiving Continuous Deep Sedation in a Hospice Care Unit
}

\author{
Hee Kyung Ahn, Ph.D., Hong Yup Ahn, Ph.D.*, So Jung Park, M.D. ${ }^{\dagger}$ and In Cheol Hwang, Ph.D. ${ }^{\ddagger}$ \\ Oncology Division, Department of Internal Medicine, Gil Medical Center, Gachon University College of Medicine, Incheon, \\ *Department of Statistics, Dongguk University, Seoul, ${ }^{\dagger}$ National Hospice Center, Goyang, \\ ${ }^{\ddagger}$ Department of Family Medicine, Gil Medical Center, Gachon University College of Medicine, Incheon, Korea
}

Continuous deep sedation (CDS) is an extreme form of palliative sedation to relieve refractory symptoms at the end of life. In this study, we shared our experiences with CDS and examined the clinical characteristics associated with survival in patients with terminal cancer who received CDS. We conducted a chart audit of 106 consecutive patients with terminal cancer who received CDS at a single hospice care unit between January 2014 and December 2016. Survival was defined as the first day of admission to the date of death. The associations between clinical characteristics and survival were presented as hazard ratios and 95\% confidence intervals using a Cox proportional hazard model. The mean age of participants was 65.2 years, and $33.0 \%(n=35)$ were women. Diazepam was the most commonly administered drug, and haloperidol or lorazepam were also used if needed. One sedative was enough for a majority of the patients. Stepwise multivariate analysis identified poor functioning, a high Palliative Prognostic Index score, hyperbilirubinemia, high serum ferritin levels, and a low number of sedatives as independent poor prognostic factors. Our experiences and findings are expected to be helpful for shared decision-making and further research on palliative sedation.

Key Words: Deep sedation, Hospices, Survival analysis, Terminal care
Received July 21, 2021

Revised September 7, 2021

Accepted September 30, 2021

\section{INTRODUCTION}

Palliative sedation is a method for decreasing awareness using strong sedatives to relieve severe pain or end-of-life symptoms that cannot be controlled with other treatments in terminally ill patients [1]. There are not yet clear clinical protocols for palliative sedation, and the protocols that are performed often vary by country, research group, or individual physician [2]. There have been multiple recent studies and accounts from different countries demonstrating such variation [3-8], but very few studies have focused on continuous deep sedation (CDS), the strongest form of palliative sedation.
In South Korea, palliative sedation was only recently added to the Clinical Practice Guideline for Care in the Last Days of Life, which is a guideline certified by the Korean Society for Hospice and Palliative Care, at recommendation level D [9]. A South Korean study on palliative sedation examined indications and the duration of sedation in 1,334 patients at a tertiary hospital who received sedation treatment in the last 2 weeks of their lives, but it mainly focused on the characteristics of medical personnel who administered sedation treatment rather than on the treatment itself [10]. Another South Korean study prospectively observed treatment indications, medications, and survival using data from 89 patients who received seda- 
tion treatment at a single hospice ward. However, the study examined the differences between intermittent and continuous sedation methods rather than the depth of sedation [11].

The aim of this study was to share our experiences administering CDS to terminal cancer patients at an in-patient hospice unit, analyze the factors related to the survival of patients who received CDS, and provide data for future decision-making and follow-up studies.

\section{METHODS}

This study used data from 106 terminal cancer patients at a single hospice care facility for whom CDS had been administered before their deaths from January 1, 2014, to December 31, 2016. CDS was defined as a maintained state of decreased consciousness to a stupor or unconsciousness caused by sedatives leading up to a patient's death [12]. This study obtained institutional review board approval from the authors' affiliated institution (GBIRB2019-309), and a retrospective review of medical records was conducted.

Sedation was administered according to a consistent pattern since patients' attending physicians did not change, and the eligibility criteria for study participants were as follows. First, patients must have had severe symptoms that did not respond to other treatment options. Refractory pain was identified after considering not only medications but also treatment choices such as palliative radiotherapy and nerve blocks. Delirium, including delirium caused by imbalances in electrolytes and medications, and dyspnea caused by conditions other than the progression of cancer, such as asthma, pneumonia, pleural effusion, pulmonary edema, and anxiety, were also considered. Symptoms were considered refractory when there was no response within a few days of administering treatment, based on the National Comprehensive Cancer Network clinical guidelines (nccn.org/guidelines/category_3).

Second, the decision to administer CDS was made through a strict decision-making process that involved a hospice team composed of medical personnel across diverse positions, family members, and the patient when possible, and all decisions were made verbally. Third, the main drug used to induce sedation was diazepam, beginning with routine $10 \mathrm{mg}$ injections throughout the day until an awareness level below a stupor was reached [13] by doubling the dose every 8 hours. If the patient did not reach an awareness level below a stupor using diazepam alone within 24 hours, another type of sedative was routinely injected in addition to diazepam. The dose titration method was the same as that of diazepam.

The following data were collected at the time of hospitalization: age, sex, primary cancer, function (Eastern Cooperative Oncology Group Performance Status [ECOG-PS] score ranging from 0 to 4) [14], Prognostic Palliative Index (PPI) score, the symptom that necessitated CDS, and the medication used to administer CDS. A blood test was conducted on the day of hospitalization according to existing protocols, and a followup test was conducted every week. When the patient was transferred from another department in the hospital and had received a blood test within the past week, a blood test was conducted after several days. The start of the survival period in this study was not the initiation of CDS but rather the beginning of in-patient hospice care, which was operationalized as the date of the first blood test in this ward. Values beginning at that point in time were used.

Statistical analysis was conducted using STATA SE 9 (STATA Corp., College Station, TX, USA). Differences in the average survival time according to participant characteristics were identified using the Kaplan-Meier survival analysis method, and Cox proportional hazard regression analysis was conducted to evaluate the factors that influenced the survival time of study participants. Using backward elimination, the final sets of variables related to the prognosis of patients were $\mathrm{ex}^{-}$ tracted, and the hazard ratio and $95 \%$ confidence interval of the variables were calculated. A P-value $<0.05$ was considered to indicate statistical significance for all analysis results.

\section{RESULTS}

The general characteristics of the participants are shown in Supplementary Table 1 . The average age of the participants was 65.2 years. A total of $33.0 \%$ of the participants were female, and most of the participants had liver, bile duct, or pancreatic cancer $(n=29)$. Patients were most often administered diazepam, followed by haloperidol (45.3\%) and lorazepam (11.3\%). Midazolam was rarely used. The average dose of each drug at the point of reaching CDS was $33.8 \mathrm{mg}$ for diaz- 
epam, $8.3 \mathrm{mg}$ for lorazepam, and $6.0 \mathrm{mg}$ for haloperidol. The most frequent indication was pain $(n=44,41.5 \%)$, followed by delirium/anxiety (39.6\%) and dyspnea (17.9\%).

The differences in survival time according to the character-

Table 1. Median Survival Time According to Patients' Characteristics upon Admission ( $\mathrm{N}=106)$.

\begin{tabular}{|c|c|c|c|c|}
\hline Variables & $\mathrm{n}$ & $\begin{array}{l}\text { MST, } \\
\text { days }\end{array}$ & $95 \% \mathrm{Cl}$ & $\mathrm{P}^{\dagger}$ \\
\hline \multicolumn{5}{|l|}{ ECOG-PS } \\
\hline $1 \sim 3$ & 75 & 13 & $11 \sim 18$ & 0.071 \\
\hline 4 & 31 & 7 & $4 \sim 12$ & \\
\hline \multicolumn{5}{|l|}{ PPI score } \\
\hline$\leq 6$ & 60 & 13 & $11 \sim 19$ & 0.023 \\
\hline$>6$ & 40 & 9 & $4 \sim 15$ & \\
\hline \multicolumn{5}{|c|}{ Leukocytosis $\left(>9.5 \times 10^{3} / \mathrm{mm}^{3}\right)$} \\
\hline No & 42 & 14.5 & 9 21 & 0.153 \\
\hline Yes & 64 & 10 & $9 \sim 13$ & \\
\hline \multicolumn{5}{|l|}{ Neutrophilia (>75\%) } \\
\hline No & 30 & 11 & 8 19 & 0.534 \\
\hline Yes & 76 & 11.5 & 9 15 & \\
\hline \multicolumn{5}{|l|}{ Lymphopenia (<20\%) } \\
\hline No & 16 & 11 & 8 22 & 0.626 \\
\hline Yes & 90 & 12 & 9 15 & \\
\hline \multicolumn{5}{|c|}{ Thrombocytopenia $\left(150 \times 10^{3} / \mathrm{mm}^{3}\right)$} \\
\hline No & 69 & 14 & $11 \sim 17$ & 0.186 \\
\hline Yes & 37 & 9 & $5 \sim 11$ & \\
\hline \multicolumn{5}{|c|}{ Hyperbilirubinemia (>1.2 mg/dL) } \\
\hline No & 71 & 13 & $10 \sim 19$ & 0.008 \\
\hline Yes & 34 & 9 & $5 \sim 13$ & \\
\hline \multicolumn{5}{|c|}{ Hypoalbuminemia (<3.5g/dL) } \\
\hline No & 27 & 18 & 8 26 & 0.218 \\
\hline Yes & 78 & 10.5 & $9 \sim 14$ & \\
\hline \multicolumn{5}{|l|}{ Azotemia (>1.2 mg/dL) } \\
\hline No & 92 & 11 & $9 \sim 15$ & 0.307 \\
\hline Yes & 13 & 10 & 4 NA & \\
\hline \multicolumn{5}{|l|}{ CRP level*, $6.58 \mathrm{mg} / \mathrm{dL}$} \\
\hline Low & 53 & 15 & $11 \sim 20$ & 0.212 \\
\hline High & 52 & 9 & $6 \sim 13$ & \\
\hline \multicolumn{5}{|c|}{ Ferritin level*, $796.5 \mathrm{ng} / \mathrm{mL}$} \\
\hline Low & 42 & 13.5 & $11 \sim 21$ & 0.006 \\
\hline High & 41 & 9 & $8 \sim 14$ & \\
\hline \multicolumn{5}{|l|}{ Indication for CDS } \\
\hline Delirium/agitation & 42 & 12 & 7 19 & 0.314 \\
\hline Dyspnea & 19 & 10 & $6 \sim 24$ & \\
\hline Pain & 44 & 11 & 9 17 & \\
\hline \multicolumn{5}{|l|}{ Drug used for CDS } \\
\hline Lorazepam & 12 & 21.5 & $15 \sim N A$ & 0.048 \\
\hline No lorazepam & 94 & 10.5 & $9 \sim 13$ & \\
\hline Haloperidol & 48 & 14.5 & 9 20 & 0.165 \\
\hline No haloperidol & 58 & 10 & $8 \sim 13$ & \\
\hline
\end{tabular}

istics of participants are shown in Table 1. Significantly longer survival times were found in those who received a PPI score of 6 or below compared to those who received a score above 6 (13 days vs. 7 days; $\mathrm{P}=0.023$ ), those who did not have hyperbilirubinemia compared to those who did (13 days vs. 9 days; $\mathrm{P}=0.008$ ), those who had low ferritin levels compared to those who had high ferritin levels (13.5 days vs. 9 days; $\mathrm{P}=0.006$ ), and those who were administered lorazepam compared to those who were not (21.5 days vs. 10.5 days; $\mathrm{P}=0.048$ ). The difference in survival time according to the number of drugs administered to induce CDS was also statistically significant $(\mathrm{P}=0.035)$.

The likelihood of a poor prognosis for those with an ECOGPS of 4 was 1.78 times higher than in those whose score was lower than 4, and a poor prognosis was 2.18 times more likely for those with a PPI score above 6 than for those with a PPI score of 6 or below. The likelihood of a poor prognosis for those who had hyperbilirubinemia was 2.04 times higher than for those who did not, and a poor prognosis was 2.28 times more likely for those who had high ferritin levels than for those who did not. The number of sedatives administered and the prognosis had a positive correlation, in which an increase in the number of sedatives administered corresponded to an increase in the likelihood of a good prognosis by 1.4 times (Table 2).

\section{DISCUSSION}

In this study, the survival time began not at the initiation of CDS but rather at the start of in-patient hospice care. The

Table 1. Continued.

\begin{tabular}{lllll}
\hline \multicolumn{1}{c}{ Variables } & $\mathrm{n}$ & $\begin{array}{l}\text { MST, } \\
\text { days }\end{array}$ & $95 \% \mathrm{Cl}$ & $\mathrm{P}^{+}$ \\
\hline No. of drugs for CDS & & & & \\
1 & 55 & 10 & $8 \sim 13$ & 0.035 \\
2 & 41 & 14 & $9 \sim 17$ & \\
$3 \& 4$ & 10 & 21.5 & $11 \sim \mathrm{NA}$ & \\
\hline
\end{tabular}

CDS: continuous deep sedation, GI: gastrointestinal, ECOG-PS: Eastern Cooperative Oncology Group Performance Status, PPI: Palliative Prognostic Index, CRP: C reactive protein, MST: median survival time, Cl: confidence interval.

*Based on the median value in the current sample. ${ }^{\dagger}$ Using the log-rank test of the Kaplan-Meier method. 
Table 2. Survival Analysis Using the Cox Proportional Hazard Model.

\begin{tabular}{|c|c|c|c|c|}
\hline \multirow{2}{*}{ Variables } & \multicolumn{2}{|c|}{ Age-and sex-adjusted } & \multicolumn{2}{|c|}{ Backward multivariate* } \\
\hline & $\mathrm{HR}(95 \% \mathrm{Cl})$ & $P$ & $\mathrm{HR}(95 \% \mathrm{Cl})$ & $P$ \\
\hline$E C O G-P S=4$ & 1.59 (1.03 2.46) & 0.038 & $1.78(1.02 \sim 3.08)$ & 0.041 \\
\hline $\mathrm{PPI}>6$ & $1.68(1.10 \sim 2.56)$ & 0.016 & $2.18(1.29 \sim 3.66)$ & 0.003 \\
\hline Leukocytosis & $1.37(0.92 \sim 2.05)$ & 0.126 & & \\
\hline Neutrophilia & $0.92(0.59 \sim 1.44)$ & 0.725 & & \\
\hline Lymphopenia & $0.91(0.53 \sim 1.56)$ & 0.721 & & \\
\hline Thrombocytopenia & $1.33(0.88 \sim 2.01)$ & 0.176 & & \\
\hline Hyperbilirubinemia & 1.90 (1.23 2.93) & 0.004 & 2.04 (1.16 3.58) & 0.013 \\
\hline Hypoalbuminemia & 1.33 (0.85 2.08) & 0.205 & & \\
\hline Azotemia & $1.37(0.76 \sim 2.46)$ & 0.299 & & \\
\hline High CRP & $1.26(0.85 \sim 1.87)$ & 0.243 & & \\
\hline High ferritin & $1.97(1.23 \sim 3.15)$ & 0.005 & $2.28(1.36 \sim 3.80)$ & 0.002 \\
\hline Dyspnea (vs. delirium) & $1.02(0.59 \sim 1.76)$ & 0.958 & & \\
\hline Pain (vs. delirium) & $0.82(0.53 \sim 1.27)$ & 0.379 & & \\
\hline Haloperidol use & $0.74(0.50 \sim 1.10)$ & 0.136 & & \\
\hline Lorazepam use & $0.55(0.30 \sim 1.02)$ & 0.057 & & \\
\hline No. of CDS drugs & $0.74(0.55 \sim 0.98)$ & 0.037 & $0.60(0.43 \sim 0.86)$ & 0.005 \\
\hline
\end{tabular}

ECOG PS: Eastern Cooperative Oncology Group Performance Status, PPI: Palliative Prognostic Index, CRP: C reactive protein, HR: hazard ratio, Cl: confidence interval, CDS: continuous deep sedation.

*Including selected variables $(P<0.05)$ in the univariate analysis.

reason for this was that it was difficult to determine the exact time of CDS initiation through retrospective medical records and that the survival time after the initiation of CDS is typically only 1 day based on the results of prior studies [11,15]. When determining the exact time of CDS initiation is difficult, the analysis can instead use information from the time of hospitalization or registration [16] and take subsequent caution when interpreting the results. Therefore, the results of this study reflect characteristics from the beginning of hospitalization related to the survival time of patients who had refractory symptoms that necessitated CDS.

In principle, palliative sedation requires progressive sedation. Of course, there continues to be controversy about the speed of sedation progression [17]. In this study, the progression from palliative sedation to CDS was not clear; however, it must be understood that the patients had refractory symptoms that necessitated CDS. The prognostic factors identified in this study (low function, high PPI scores, hyperbilirubinemia, high blood ferritin levels, and others) have already been previously identified as prognostic factors in terminal cancer patients. For patients with highly severe symptoms, the significance of those factors was considered to have been maintained. Administer- ing a higher number of sedatives indicated that it took more time to reach CDS, thus also increasing the patient's recorded survival time.

The sedatives used for palliative sedation have their strengths and limitations [18]. Midazolam is fast-acting, but the range of responses varies by patient, and while the patient response to lorazepam tends to have less variation than midazolam, more time is needed to reach maximum effectiveness when administering lorazepam. Diazepam, which was the primary drug used in this study, reaches its maximum effectiveness quickly, but when it is injected over a long period of time, the sedative effect from its metabolites is cumulative. This limitation, however, can make it a stable and effective sedative in the context of a hospice ward where the need is mainly for irreversible sedation.

This study has the following limitations. First, since the study retrospectively reviewed medical records, data on the continuous evaluation of symptoms and important information including changes in consciousness after sedation that could have affected the results were not obtained. Moreover, since there was no control group composed of individuals who did not receive CDS, the study could not examine the effect of 
CDS itself on survival. Second, since the study was based on experiences within a single institution, the generalizability of the findings is limited. It is possible that different trends would be observed in primary and secondary hospice hospitals or at other types of hospice facilities. Third, various factors that can influence sedation, including the socioeconomic background of patients and family members, were not included in the analysis. Despite these limitations, this study provides valuable data about CDS in the context of in-patient hospice care. As public interest in end-of-life care increases following the implementation of South Korea's Act on Decisions on Life-Sustaining Treatment, it is expected that end-of-life guidelines suited to a South Korean context will be developed based on the results of various studies.

\section{CONFLICT OF INTEREST}

No potential conflict of interest relevant to this article was reported.

\section{ORCID}

Hee Kyung Ahn, https://orcid.org/0000-0001-7786-7233

Hong Yup Ahn, https://orcid.org/0000-0001-8149-6981

So Jung Park, https://orcid.org/0000-0002-5444-0250

In Cheol Hwang, https://orcid.org/0000-0003-4677-5525

\section{AUTHOR'S CONTRIBUTIONS}

Conception or design of the work: ICH. Data collection: HKA, ICH. Data analysis and interpretation: all authors. Drafting the article: HKA. Critical revision of the article: HYA, ICH. Final approval of the version to be published: all authors.

\section{SUPPLEMENTARY MATERIALS}

Supplementary materials can be found via https://doi. org/10.14475/jhpc.2021.24.4.254.

\section{REFERENCES}

1. Kim DS. Terminal sedation. Korean J Hosp Palliat Care 2010;13:139-42.

2. Maltoni M, Scarpi E, Nanni O. Palliative sedation for intolerable suffering. Curr Opin Oncol 2014;26:389-94.

3. Gu X, Cheng W, Chen M, Liu M, Zhang Z. Palliative sedation for terminally ill cancer patients in a tertiary cancer center in Shanghai, China. BMC Palliat Care 2015;14:5.

4. Schur S, Weixler D, Gabl C, Kreye G, Likar R, Masel EK, et al. Sedation at the end of life - a nation-wide study in palliative care units in Austria. BMC Palliat Care 2016;15:50.

5. Caraceni A, Speranza R, Spoldi E, Ambroset CS, Canestrari S, Marinari M, et al. Palliative sedation in terminal cancer patients admitted to hospice or home care programs: does the setting matter? Results from a national multicenter observational study. J Pain Symptom Manage 2018;56:33-43.

6. Parra Palacio S, Giraldo Hoyos CE, Arias Rodriguez C, Mejia Arrieta D, Vargas Gomez JJ, Krikorian A. Palliative sedation in advanced cancer patients hospitalized in a specialized palliative care unit. Support Care Cancer 2018;26:3173-80.

7. Prado BL, Gomes DBD, Uson Junior PLS, Taranto P, Franca MS, Eiger D, et al. Continuous palliative sedation for patients with advanced cancer at a tertiary care cancer center. BMC Palliat Care 2018;17:13.

8. Tin WW, Lo SH, Wong FC. A retrospective review for the use of palliative sedation in a regional hospital in Hong Kong. Ann Palliat Med 2019.

9. Shin J, Chang YJ, Park S, Choi JY, Kim S, Choi YS, et al. Clinical practice guideline care for last days of life. Korean J Hosp Palliat Care 2020;23:103-13.

10. Kim YS, Song HN, Ahn JS, Koh SJ, Ji JH, Hwang IG, et al. Sedation for terminally ill cancer patients: a multicenter retrospective cohort study in South Korea. Medicine (Baltimore) 2019;98:e14278.

11. Won YW, Chun HS, Seo M, Kim RB, Kim JH, Kang JH. Clinical patterns of continuous and intermittent palliative sedation in patients with terminal cancer: a descriptive, observational study. J Pain Symptom Manage 2019;58:65-71.

12. Park SJ, Ahn HK, Ahn HY, Han KT, Hwang IC. Association between continuous deep sedation and survival time in terminally ill cancer patients. Support Care Cancer 2021;29:525-31.

13. Morita T, Imai K, Yokomichi N, Mori M, Kizawa Y, Tsuneto S. Continuous deep sedation: a proposal for performing more rigorous empirical research. J Pain Symptom Manage 2017;53:146-52.

14. Oken MM, Creech RH, Tormey DC, Horton J, Davis TE, McFadden ET, et al. Toxicity and response criteria of the Eastern Cooperative Oncol- 
ogy Group. Am J Clin Oncol 1982;5:649-55.

15. Maltoni M, Pittureri C, Scarpi E, Piccinini L, Martini F, Turci P, et al. Palliative sedation therapy does not hasten death: results from a prospective multicenter study. Ann Oncol 2009;20:1163-9.

16. Maeda I, Morita T, Yamaguchi T, Inoue S, Ikenaga M, Matsumoto Y, et al. Effect of continuous deep sedation on survival in patients with advanced cancer (J-Proval): a propensity score-weighted analysis of a prospective cohort study. Lancet Oncol 2016;17:115-22.

17. Maltoni M, Setola E. Palliative sedation in patients with cancer. Cancer Control 2015;22:433-41.

18. Bodnar J. A review of agents for palliative sedation/continuous deep sedation: pharmacology and practical applications. J Pain Palliat Care Pharmacother 2017;31:16-37. 
Hee Kyung Ahn, et al

Supplementary Table 1. Patient Characteristics $(\mathrm{N}=106)$.

\begin{tabular}{|c|c|c|}
\hline Characteristics & Total (\%) & Mean \pm SD \\
\hline Age (yr) & & $65.2 \pm 12.8$ \\
\hline \multicolumn{3}{|l|}{ Sex } \\
\hline Female & $35(33.0)$ & \\
\hline \multicolumn{3}{|l|}{ Primary cancer site } \\
\hline Hepato-Biliary-Pancreatic & $29(27.4)$ & \\
\hline $\begin{array}{l}\text { Gastrointestinal } \\
\text { (esophagus, stomach, colorectal) }\end{array}$ & $24(22.6)$ & \\
\hline Lung & $20(18.9)$ & \\
\hline $\begin{array}{l}\text { Genitourinary } \\
\text { (bladder, prostate, cervix, ovary, endometrial) }\end{array}$ & $13(12.3)$ & \\
\hline Breast & $11(10.4)$ & \\
\hline Other & $9(8.5)$ & \\
\hline Drugs for CDS & & Maintenance dose \\
\hline Diazepam & 105 (99.1) & $33.8 \pm 20.6$ \\
\hline Haloperidol & $48(45.3)$ & $6.0 \pm 2.1$ \\
\hline Lorazepam & $12(11.3)$ & $8.3 \pm 5.0$ \\
\hline Midazolam & $2(1.9)$ & \\
\hline \multicolumn{3}{|l|}{ Indication for CDS } \\
\hline Pain & $44(41.5)$ & \\
\hline Delirium/agitation & $42(39.6)$ & \\
\hline Dyspnea & $19(17.9)$ & \\
\hline Others & $1(0.9)$ & \\
\hline
\end{tabular}

Data are presented as number (\%) or mean \pm SD.

SD: standard deviation, CDS: continuous deep sedation. 